\title{
Strategies for activating TLR4 to improve vaccine effectiveness
}

\author{
BoYoon Chang ${ }^{1}$ and SungYeon Kim ${ }^{1}$ \\ ${ }^{1}$ Wonkwang University College of Pharmacy
}

September 22, 2020

\begin{abstract}
Background and Purpose: In the development of vaccines, it is important to increase the antigen of the vaccine. We have confirmed the potential of Morus alba L., a Toll-like receptor 4 (TLR4) activator, as an adjuvant as a method for increasing the antigenicity of the vaccine. Experimental Approach: TLR4 is highly expressed on immune cells, therefore, the present study was undertaken to investigate the underlying immunological processes of Morus alba L. as an oral adjuvant in a mouse model. Mice were immunized with two types of antigen (ovalbumin (OVA) or inactivated influenza vaccine) combined with Morus alba L. Key Results: In the OVA antigen model, the co-administration of Morus alba L. significantly promoted phagocytosis in murine peritoneal macrophages. The maturation and function of dendritic cells were significantly up-regulated the expression of MHC-II, CD86, and CD80 compared to OVA alone. The titers of OVA-specific IgG, IgG subtype responses, and IgA were increased, the proliferation of $\mathrm{T}$ and B-cells was markedly enhanced, and the production of IL-12, IFN-?, and IL-4 was better than OVA alone. In the inactivated influenza H1N1(PR8) vaccine model, the co-administration of Morus alba $\mathrm{L}$. with vaccine significantly increased the $\operatorname{IgM}, \operatorname{IgG}$, and IgG subtype responses. The immunization of mice with Morus alba L. adjuvant reduced mortality in mice after a lethal challenge with influenza virus. Conclusion and Implications: Morus alba L. is a novel mucosal adjuvant for vaccination that provided safe and effective adjuvant effects and successfully induced both serum and mucosal antibody responses and cell-mediated responses.
\end{abstract}

\section{Introduction}

The 2019 novel coronavirus (COVID-19) has faced global pandemic. This is different from the past Severe Acute Respiratory Syndrome(SARS-CoV) and Middle East Respiratory Syndrome coronavirus (MERS$\mathrm{CoV}$ ), and may suggest that the COVID-19 situation may not be easily terminated through the second and third wave. Various strategies have been applied to control the rapidly expanding COVID-19 (Kannan, Shaik Syed Ali, Sheeza, \& Hemalatha, 2020; Singhal, 2020). There is a need for the development of vaccines that allow the formation of antibodies capable of controlling COVID-19, which is why global pharmaceutical companies are in the process of clinical research on vaccines (L. Yang, Tian, \& Liu, 2020). Vaccine development requires three technologies, an antigen, a vaccine adjuvant, and delivery technology. Antigen-related technologies relate to antigen design and production techniques that induce protective immune responses. Vaccine adjuvants are designed to maintain high protective immune responses to the antigen over long periods of time. Finally, the vaccine delivery technology determines the vaccination route $\{$ Ahmed, 2011 \#1\}. Presently, vaccine development has mainly focused on antigen development (Calzas \& Chevalier, 2019; Martinon, Cisneros, \& Villicana, 2019; Mohan, Zhu, Wang, \& Wang, 2018). However, the importance of immunity-enhancing vaccine adjuvants has been emphasized to develop new preventive vaccines against infectious diseases or to improve the efficacy of present vaccines (Roy et al., 2014; Rydell \& Sjoholm, 2004). The use of adjuvants may increase the long-term immunogenicity of the vaccine and thus, reduce or eliminate the need for additional inoculations (Ahmed, Plotkin, Black, \& Coffman, 2011). Moreover, since immunity-enhancers tends to increase the cross-reactivity of the vaccine, it can also increase the protective effect against serotype strains not included in the vaccine. If a vaccine, such as an influenza vaccine, is temporarily needed due to a global pandemic, it is also possible to increase the vaccine supply by lowering 
the antigen dose(Ahmed et al., 2011; Cribbs et al., 2003).

Immune cells generate a specific immune response to each pathogenic bacterium or virus at the time of infection but they also have receptors called pattern recognition receptors (PRRs) that respond to commonlyencountered molecules, such as bacterial cell walls, LPS, proteins or viral RNA and DNA (Jahanban-Esfahlan, Seidi, \& Majidinia, 2019; Joshi, Shaw, \& Quagliarello, 2009). The Toll-like receptor (TLR) is a typical PRR and 11 species are known in humans. TLR agonists are highly active against immune cells and are being developed as vaccine adjuvants (Medvedev, 2013).

Polysaccharides derived from natural products have potential as immunomodulators because they are large molecules that affect the immune system and they have been widely used in clinical applications. Most polysaccharides present in natural products are non-toxic and do not cause the serious side effects associated with other bacterial polysaccharides or synthetic substances that are capable of immunomodulation. They also maintain homeostasis in the body (Harandi, 2018). Recent studies have shown that polysaccharides present in natural products exert immunosuppressive effects on antigens and vaccines in both cellular and humoral immunity, making them alternatives to conventional aluminum salts as vaccine adjuvants for vaccines (Liu et al., 2019; Tian \& Liang, 2019). Specifically, plant polysaccharides can safely induce humoral immunity, as well as cellular immunity, as vaccine adjuvants. Their advantages are availability at relatively low cost and application to a wide range of vaccines against cancer and infectious diseases (Gu et al., 2019; Munoz, Porsch, \& St Geme, 2019; Tian \& Liang, 2019).

Morus alba $L$. is enriched in polysaccharides, anthocyanins, alkaloids, and flavonoids. Pharmacological functions attributed to $M$. alba L. include anti-oxidative, anti-inflammatory, anti-diabetic, as well as cardiovascular, hepatoprotective, and neuroprotective activities (Hwang \& Kim, 2004; H. G. Kim et al., 2010). M. alba L. has been found to be immunostimulatory in vitroand in vivo. We also found that when M. alba L. was used in combination with anticancer drugs, such as fluorouracil (5FU), it decreased the side effects of the anticancer drug and increased immune function. We recently found that $M$. alba L. can activate Toll-like receptor 4 (TLR4) on antigen-presenting cells (APCs) (Chang, Kim, Lee, Park, \& Kim, 2015; S. B. Kim et al., 2013; X. Y. Yang et al., 2009). However, the oral administration of M. alba L. to improve immunogenicity as a vaccine adjuvant has been rarely reported. If effective, the ingestion of the $M$. alba L. would be more a more readily accepted and convenient application than intramuscular or nasal administration.

In this study, we conducted studies in a mouse model to investigate the effects of orally administered $M$. alba L. on modulating the efficacy of ovalbumin and PR8 influenza vaccine immunizations.

\section{Materials and methods}

\section{Animals}

Female Balb/c mice (6 weeks old , 18.1 $\pm 1.4 \mathrm{~g}$ ) were housed in specific pathogen-free (SPF) conditions at 21 to $24{ }^{\circ} \mathrm{C}$ and $40 \%$ to $60 \%$ relative humidity with a $12 \mathrm{~h}$ light-dark cycle. All the animals were acclimatized for at least one week prior to the start of the experiments. All studies were performed in accordance with the guidelines for animal experimentation by Wonkwang University and were approved by the university's Institutional Animal Care and Use Committee (Approval No. WKU13-50, WKU18-43).

\section{Madin-Darby canine kidney cells}

Madin-Darby canine kidney (MDCK, American Type Culture Collection(ATCC), Manassas, VA, USA) cells were cultured in monolayers in minimum essential medium (MEM, Gibco) supplemented with 10\% heatinactivated fetal calf serum (FCS).

\section{Virus strains and HA vaccine}

The type A influenza viruses used in this study were the mouse-adapted A/Puerto Rico/8/1934 (H1N1) [PR8] viral strains obtained from ATCC. Type A influenza viruses were propagated in MDCK cells in serum-free media in the presence of TPCK-trypsin and the median tissue culture infective dose $\left(\mathrm{TCID}_{50}\right)$ and median lethal dose $\left(\mathrm{LD}_{50}\right)$ of the viruses were calculated. The HA vaccines (viral vaccines) were prepared from 
influenza viruses, including A/Puerto Rico/8/1934 (A/PR8:H1N1) strains, according to the method of Jian et al . Briefly, we performed a plaque assay with MDCK and a hemagglutination assay with serum to calculate the titer of virus stock. The titer of virus stock showed that 1 hemagglutination unit (HAU) was equal to $10^{3} \mathrm{PFU}$. The PR8 virus stock were exposed to ultraviolet light (UV) for 45 minutes.(Jian, Chang, Lin, Yang, \& Chuang, 2013) The loss of infectivity of the UV-inactivated PR8 virus was confirmed by the plaque assay.

\section{Extract of Morus alba fruits}

The dried fruits of M. alba L. were purchased from a local herbal market in Jeonbuk, Korea. The fruits were pulverized into powder and extracted twice with hot water $(70 @ \mathrm{C})$ for 3 hours. The solvent was removed under reduced pressure in an RV10 rotary evaporator (IKA, Staufen, Germany) to yield an M. alba fruit hot water extract $(59.3 \%, \mathrm{w} / \mathrm{w})$. The extract was dried to a powder and kept in a closed container until use. To avoid variations in activity for different preparations, enough extract was obtained in one batch for use throughout the experiments. The content of the marker pyrrole alkaloid, 2-formyl-5-(methoxymethyl)- $1 \mathrm{H}$ -pyrrole-1-butanoic acid, inMorus alba L. was quantitated using high-performance liquid chromatography (HPLC) (Chang et al., 2015).

\section{Immunization}

Female Balb/c mice (6 weeks old , 18.1 $\pm 1.4 \mathrm{~g}$ ) were randomly divided into five groups ( $\mathrm{n}=10$ per group): normal, OVA, OVA+ M. alba L. $100 \mathrm{mg} / \mathrm{kg}$, OVA+ M. alba L. $300 \mathrm{mg} / \mathrm{kg}$, and OVA+ CT $0.5 \mathrm{mg} / \mathrm{kg}$ groups as described above. Mice were orally immunized four times at weekly intervals (days 0, 7, 14, and 21) with $10 \mathrm{mg} / \mathrm{kg}$ of free OVA or OVA mixed with 100 or $300 \mathrm{mg} / \mathrm{kg} / 10 \mathrm{~mL}$ of Morus alba L. dissolved in phosphatebuffered saline (PBS). The positive control mice were immunized with OVA mixed with $0.5 \mathrm{mg} / \mathrm{kg} / 5 \mathrm{~mL}$ $\mathrm{CT}$ (cholea toxin) (Fig 1A).

Another female Balb/c mice (6 weeks old , 18.1 $1.4 \mathrm{~g}$ ) were randomly divided into four groups ( $\mathrm{n}=10$ per group): normal, control, vaccine, vaccine + M. alba L. $100 \mathrm{mg} / \mathrm{kg}$, and OVA+ Alum $0.5 \mathrm{mg} / \mathrm{kg}$ groups as described above. Mice received $\mathrm{H} 1 \mathrm{~N} 1$ vaccine $(12 \mathrm{HAU} / 100 \mathrm{uL})$ administered twice, at a 0 and 14 days, in 100 ?L volume by intramuscular injection. The vaccine was diluted in saline to achieve the desired dose and mixed with alum (positive control) in a 1:1 ratio. The mice were orally immunized with $100 \mathrm{mg} / \mathrm{kg}$ Morus alba L. four times at weekly intervals (days 0, 7, 14, and 21) in PBS. The control mice were given PBS (Fig 1B).

\section{Influenza Challenges}

Four weeks after immunization, the mice were challenged intranasally with mouse-adapted influenza A/Puerto Rico/8/34 (PR8; H1N1). Groups of 10 mice were challenged intranasally (i.n.) on day 28. The mice were anesthetized intraperitoneally with ketamine/xylazine (2 mg ketamine and $0.15 \mathrm{mg}$ xylazine) before i.n. infection with $10 \mu \mathrm{L}$ of sterile saline containing PR8 (20 HAU/mouse). The protective efficacy of the immunization was measured by survival up to 14 days and mice that lost [?] $25 \%$ of their initial body weight was euthanized according to institutional guidelines.

\section{Sample collection}

To determine the antibody $(\mathrm{Ab})$ responses, the mice were bled a week after immunization and the serum was collected. Saliva sample was collected with pipetting method. Saliva samples harvested that PBS placed in the oral cavity at 3 minutes later, transferred to a microcentrifuge tube. For intestinal IgA, the large intestine was excised $5 \mathrm{~cm}$ (from the rectum), The saliva and, intestinal fluid from the entire gut was collected, mixed with cold saline $\left(4{ }^{\circ} \mathrm{C}\right)$ and centrifuged for $10 \mathrm{~min}$ at $2000 \times \mathrm{g}$. The supernatants were collected and frozen. Peritoneal exudate cells were collected by intraperitoneal injection with $10 \mathrm{~mL}$ of DMEM medium. The cells were centrifuged at $2500 \mathrm{rpm}$ for $10 \mathrm{~min}$ and the supernatants were discarded. These were resuspended in DMEM complete medium. Splenocytes were prepared from the spleens by chopping on sterile glass sides and centrifugation at $1400 \mathrm{rpm}$ for $5 \mathrm{~min}$. The erythrocytes were lysed with $1 \mathrm{~mL}$ of ammonium chloride $(0.8$ $\% \mathrm{w} / \mathrm{v}$ ). After centrifugation (1400 rpm for $5 \mathrm{~min}$ ), $10 \mathrm{~mL}$ of medium was added to the pelleted cells and 
filtered through a fine cell strainer (BD Falcon, BD Bioscience) of $40 \mu \mathrm{m}$ pore size. The cell numbers and viability were assessed microscopically by the trypan blue dye exclusion technique (Xu et al., 2009).

\section{Phagocytic activity of macrophages}

To evaluate neutral red phagocytosis by peritoneal macrophages, $100 \mu \mathrm{l}$ of $0.075 \%$ aseptic neutral red solution was added and incubated for $1 \mathrm{~h}$. The supernatant was discarded and the cells were washed twice with PBS. Then, $100 \mu \mathrm{l}$ of cell lysate solution (ethanol $50 \%$ and $1 \%$ acetic acid at the ratio of $1: 1$ ) was added to a 96 -well plate to lyse the cells at room temperature for $2 \mathrm{~h}$. The optical density at $540 \mathrm{~nm}$ was measured by ELISA reader.

\section{Flow cytometry}

Splenocytes were incubated for 15 min with an anti-CD16/32 monoclonal antibody (mAb) to prevent the nonspecific binding of antibodies and were then reacted with primary mAb on ice for $30 \mathrm{~min}$. The following mAbs were used in this study: FITC-conjugated anti-MHCII, PE-conjugated anti-CD80/86, and PE-Cy7 conjugated anti-F4/80. All flow cytometry data were acquired and analyzed with a FACS Calibur flow cytometer.

\section{Detection of antibody responses}

Antigen-specific IgG, IgG1, IgG2a, IgE, and IgA antibodies in the sera were detected by indirect ELISA as previously described (Huber et al., 2006). The absorbance was measured with an ELISA reader at $490 \mathrm{~nm}$. The data are expressed as the mean absorbance of the samples minus the mean absorbance of the normal control.

\section{Cytokines}

Cytokine concentrations in the serum were measured by ELISA as previously described (Chang et al., 2015; Jones, Ackermann, Wille, Hunter, \& Scott, 2002). Purified anti-mouse capture and biotinylated detection of Abs to IL-12p70, IFN- $\gamma$, IL-4 were conducted. All Abs were obtained from BD Biosciences.

\section{Measurement of viral titers in the mouse lung tissues}

Mouse lung tissue samples were collected four days after the viral challenge, homogenized in PBS containing antibiotics, and centrifuged at $12,000 \times \mathrm{g}$. The supernatants were 10 -fold serially diluted, inoculated into $95 \%$ semi-confluent MDCK cells, and incubated at $37^{\circ} \mathrm{C}$ for $1 \mathrm{~h}$. The culture media were then discarded, a low-temperature melting agarose solution was added over the cells, and the cells were incubated at $37^{\circ} \mathrm{C}$ for three days. The cells were then fixed with $4 \%$ paraformaldehyde, stained with crystal violet, and the viral plaques formed were counted.

\section{Data analysis}

The data and statistical analysis comply with the recommendations of the British Journal of Pharmacology on experimental design and analysis in pharmacology (Curtis et al., 2018). Sample sizes subjected to statistical analysis at least $n=10$, where $n=$ number of independent values. Data are expressed as the mean $\pm S D$, and statistical significance was determined by analysis of variance and Student's $t$-tests. $P$-values $<0.05$ were considered statistically significant.

\section{Results}

\section{OVA immunization model}

\section{Body and splenic weights}

The body weights of the animals were carefully monitored. Only slight changes in the weights from Day 0 to Day 28 were seen and may be attributed to differences in the metabolic activity of the animals (data not shown). The spleen weights (collected on Day 28) were measured to assess their in vivo immunomodulatory 
status following administration of antigen (OVA) in the presence or absence ofMorus alba L. or CT treatments. A significant increase in the spleen weights was seen following treatment with OVA and Morus alba L. or CT and Morus alba L. compared to the OVA alone group (Fig 2A).

Peritoneal macrophage activity

To study the ability of Morus alba L. to stimulate the immune response, we examined the activity of peritoneal macrophages obtained from mice $60 \mathrm{~min}$ after the fourth immunization. The phagocytotic activity Like CT, the Morus alba L. combination group/of peritoneal macrophages from mice treated with OVA or CT plus Morus alba $\mathrm{L}$. was higher than that of the OVA alone group (Fig 2A).

Immune modulator molecules

MHC-II, CD80, and CD86 are representative co-stimulatory molecules that provide the second signal to develop an effective immune response and support T-cell activation (Banchereau \& Steinman, 1998). The expression of MHC-II, CD80, and CD86 on splenic macrophages was significantly upregulated by Morus alba L. treatment in combination with OVA or CT in a dose-dependent manner. As shown in Fig. 2B, the stimulation of splenic macrophages by the combination of Morus alba L. and OVA or CT resulted in significant upregulations of co-stimulatory molecules (CD80 and CD86) and major histocompatibility complex (MHC) II molecules compared to the OVA alone group $(p<0.05)$.

Antigen-specific antibodies

The titer of the serum anti-OVA IgG Abs was increased in the groups treated with Morus alba L. and OVA or CT compared to the OVA-only treated group (Fig. 3). A comparable immune response was produced when an antigen mixed with CT was used as a positive control. To determine the immunoglobulin patterns induced by oral Morus alba L. administration, OVA-specific serum IgG1 and IgG2a anti-OVA Abs were measured. The oral immunization of OVA and CT induced a predominantly antigen-specific IgG1 response, with little IgG2a. Co-administration of OVA with Morus alba L. increased the levels of both the IgG1 and IgG2a Abs two-fold (Fig. $3 \mathrm{C}, \mathrm{D}$ ). No change in the IgE content was observed. CT induced the secretion of IgE Abs two-fold compared to OVA (Fig. 3E).

We further determined whether oral tolerance affected the mucosal Ab responses in serum, saliva, and the intestine. The oral immunization of OVA plus CT as an adjuvant elicited significant antigen-specific mucosal IgA Ab responses. The serum and intestinal responses were significantly increased in the Morus alba L. or CT combination groups compared to the OVA group (Fig. $3 \mathrm{~F}, \mathrm{H}$ ). There was no effect on salivary IgA responses (Fig. 3G).

Cytokines

As illustrated in Figure 4, the mice that received 100 or $300 \mathrm{mg} / \mathrm{kg}$ Morus alba L. showed statistically significant enhancement in Th1 and Th2-related cytokines, including IFN- $\gamma$, IL-12, and IL-4. In contrast, the concentration of Th1 cytokines, including IFN- $\gamma$ and IL-12, in the CT group was similar to the OVA group.

\section{HA vaccine immunization model}

\section{Body Weight}

No weight change was observed from administration of the vaccine and vaccine adjuvant during the immunization period. However, after infection with influenza virus, a weight loss of $35.4 \%$ was observed in the influenza virus-infected group, whereas, the Morus alba L. plus adjuvant group experienced an $8.6 \%$ weight loss (Fig. 6A).

Antigen-specific antibodies

Serum-specific IgG and the IgM were measured by an indirect ELISA technique to evaluate the adjuvant effect of Morus alba L. on humoral immune responses. The highest igM values were obtained one week after the 
viral challenges. In contrast, the IgG levels showed a tendency to increase steadily after the viral challenges. The IgM and IgG levels in vaccinated mice administered Morus alba L. were higher than those given vaccine only. Based on the above results, antibody isotypes and IgG subclasses were measured in sera collected from individual mice on day 28. The typical Th2 immune response anti-OVA IgG1 concentrations were the highest in the Morus alba L. and alum groups. However, the Th1 immune response IgG2a concentrations were decreased in alum-treated mice compared to Morus alba L. treatment. Morus alba L. treatment increased IgG2a concentrations compared to the vaccinated group (Fig. 5).

\section{Mortality}

To evaluate the potency of Morus alba L.-induced immune responses against a lethal infection with influenza virus, vaccinated mice were challenged with mouse-adapted H1N1 influenza A subtypes. The protective efficacies were measured by survival rates monitored every day for 14 days. As shown in Fig. 6B, Morus alba L. adjuvants enhanced mouse survival rates post-challenge with a single-dose of HA vaccine. The protective efficacy of the non-adjuvanted vaccine was only $50 \%$. However, mice given Morus alba L. adjuvant were protected against the viral challenge.

\section{Discussion}

Vaccine adjuvants are represented by different classes of compounds that display adjuvant activity in preclinical models. Among them, microbial products, mineral salts, emulsions, microparticles, nucleic acids, small molecules, saponins, and liposomes, exert their function by diverse and often, poorly characterized mechanisms of action. However, only a few of them have been licensed for human use, while the vast majority failed to demonstrate an unacceptable safety profile.

An efficient adjuvant should have minimal toxicity and evoke a powerful humoral or/and cellular immune response against the specific antigen (Kohn et al., 1969). Our previous studies indicated that the extracts and components of a Morus alba L fruit water extract were safe (Chang, Kim, Lee, Park, \& Kim, 2016), immunostimulatory (Chang et al., 2015; S. B. Kim, Chang, Hwang, Kim, \& Lee, 2014; S. B. Kim et al., 2013; $\mathrm{X}$. Y. Yang et al., 2009), and had indirect anti-cancer activity by enhancing the immune responses mediated by TLR4 signaling. TLR4 is expressed on macrophages, dendritic cells, B-cells, T-cells, and endothelial cells. The role of TLR as an M. alba L. receptor was clearly demonstrated in our previous study (Chang et al., 2015). The binding of TLR4 to M. alba L. activated signaling pathways, including MAPKs and NF- $x \mathrm{~B}$. The activation of MAPKs is required for the induction of nitric oxide (NO) as it controls the activation of NF- $x$ B. In this study, the role of TLR4 as an M. alba L receptor was confirmed in macrophages (Chang et al., 2015; X. Y. Yang et al., 2009).

The immune adjuvant property of Morus alba L. was investigated using Morus alba L. as a weak antigen in a Balb/c mice animal model. OVA is a well-known model antigen with weak immunogenic properties (Roy et al., 2014; Wusiman et al., 2019). In the present study, Morus alba L. was assessed for its potential adjuvant properties. This study showed that Morus alba L. increased serum antibody titers up to those induced by $\mathrm{CT}$, the most powerful modern adjuvant, used as a positive control (Terrinoni, Holmgren, Lebens, \& Larena, 2019). To characterized the nature of the immune responses enhanced byMorus alba L., we analyzed the serum IgG subclass patterns. In the absence of cytokine secretion data, IgG isotypes are commonly used as an indirect means of determining the Th-bias of induced immune responses. It is generally accepted that an IgG1 response is induced with help from Th2 cells, whereas Th1 activity leads to the additional production of IgG2a Abs. OVA alone was found to predominantly enhance specific IgG1 Abs. Consistent with previous reports, oral immunization with OVA and CT induced robust IgG1 and low IgG2a Ab responses.

The co-administration of OVA with Morus alba L. was used to increase levels of both IgG1 and IgG2a. The data obtained indicated that Morus alba L. stimulated the humoral response without effecting the $\operatorname{IgG} 1 / \operatorname{IgG} 2 \mathrm{a}$ titer ratios. Dual IgG1 and IgG2a-potentiating activity has been reported for QS-21, polyacryl starch microparticles, and a pectic polysaccharide of Lemnan minor L. (Cribbs et al., 2003; Popov et al., 2006; Rydell \& Sjoholm, 2004). The strong systemic cellular response demonstrated by the IgG2a titers showed that the Th1 profiles were increased by Morus alba L. Antibody responses were detected in mice immunized 
with OVA and Morus albaL. However, Morus alba L. failed to change serum OVA-specific IgE titers.

Macrophages, which function as antigen-presenting cells for T-cells, have evolved a set of non-clonal pattern recognition receptors that bind carbohydrate polymers. Morus alba L. may, therefore, stimulate macrophages by mimicking natural danger signals provided by bacteria and viruses (Ghorpade, Holla, Sinha, Alagesan, \& Balaji, 2013; Medvedev, 2013). CT has previously been shown to enhance the cell surface expression of MHC antigens, co-stimulatory molecules, and chemokine receptors on macrophages and to affect the secretion of cytokines, such as IL-12 and TNF- $\alpha$ (Roy et al., 2014). We also found a strong upregulation of co-stimulatory molecules, MHCII, CD80, and CD86. Cytokines have pivotal functions in regulating immune responses. Th2 lymphocyte cytokines, such as IL- 4, IL-5, and IL-10 can augment IgG1 production, while IL-12, TNF- $\alpha$, and IFN- $\gamma$ produced by Th1 lymphocytes can improve IgG2a production (Cribbs et al., 2003; Jones et al., 2002; Tuo, Palmer, McGuire, Zhu, \& Brown, 2000). The higher level of all IgG subclasses may be explained by increased IL-4, IL-12 and IFN- $\gamma$ production, suggesting that Morus alba L. can enhance both Th1 and Th2 immune responses. Bacterial toxins, such as CT are potentially too toxic for use in humans. In contrast, Morus alba L. has been administered to humans by a variety of routes without toxic effects.

Mucosal tissues are the main entry portal of many pathogens, including influenza, and the mucosal immune system provides the first line of defense against infections, apart from innate immunity (Akter et al., 2019; Rydell \& Sjoholm, 2004). Immunization with Morus alba L. in the vaccinated group increased IgM and IgG more than vaccination only.

The immune reaction to inactivated influenza virus is characterized by IgG2a and IgG1 responses. Particulate adjuvants, such as alum or M59, may stimulate immune responses to IgG1, while some TLR agonists may stimulate IgG2a responses (Goff et al., 2017; Jian et al., 2013; Yanase et al., 2014). In comparisons between Morus alba L., alum, and non-adjuvant vaccines conducted in the present study, significant differences in IgG-subtypes were observed. Morus alba L. induced higher levels of IgG1 and IgG2a antibodies than HA vaccines. In contrast, alum increased IgG1 antibody levels similar to those of Morus alba L. but did not elicit an appropriate IgG2a response. Indeed, alum attenuated the IgG2a response induced by HA vaccines.

Influenza virus vaccines are largely effective only against a matched strain, necessitating accurate prediction of the upcoming epidemic strains during annual vaccine reformulation. By enhancing vaccine immunogenicity and improving the quality and persistence of the immune response, adjuvants may provide long-lasting efficacy against drifted strains (Huber et al., 2006; Joshi et al., 2009). Indeed, whenMorus alba L. was administered with the HA vaccine, it provided a protective immune response against a lethal challenge with the H1N1 virus.

In the last decade, experimental adjuvants for viral vaccines have proliferated. While many have demonstrated efficacy in animal models, few have been able to move through the regulatory hurdles because of safety and reactogenicity concerns (Kuznetsova \& Persiyanova, 2019). Morus alba L., with a variety of antigens, induced rapid and cross-reactive humoral and cellular immunity to elicit broad protection. Morus alba L., which has increased antigenicity and favorable safety, has confirmed its potential as an oral adjuvant for a variety of influenza virus vaccines, as well as an immunopotentiator.

Author contributions: B.Y.C, and S.Y.K. designed the experiments. B.Y.C, and S.Y.K. performed the experiments. B.Y.C, and S.Y.K. drafted the manuscript; and all the authors revised, edited, and approved the final version of the manuscript.

Acknowledgments: This work was supported by Wonkwangs University in 2020.

Conflict of interest: The authors declare no conflicts of interest.

Declaration of transparency and scientific rigour: This Declaration acknowledges that this paper adheres to the principles for transparent reporting and scientific rigour of preclinical research as stated in the BJP guidelines for Design \& Analysis, and Animal Experimentation and as recommended by funding agencies, publishers and other organizations engaged with supporting research. 


\section{Reference}

Ahmed, S. S., Plotkin, S. A., Black, S., \& Coffman, R. L. (2011). Assessing the safety of adjuvanted vaccines. Sci Transl Med, 3 (93), 93rv92. doi: 10.1126/scitranslmed.3002302

Akter, A., Dash, P., Aktar, A., Jahan, S. R., Afrin, S., Basher, S. R., . . . Qadri, F. (2019). Induction of systemic, mucosal and memory antibody responses targeting Vibrio cholerae O1 O-specific polysaccharide (OSP) in adults following oral vaccination with an oral killed whole cell cholera vaccine in Bangladesh. 13 (8), e0007634. doi: 10.1371/journal.pntd.0007634

Banchereau, J., \& Steinman, R. M. (1998). Dendritic cells and the control of immunity. Nature, 392 (6673), 245-252. doi: $10.1038 / 32588$

Calzas, C., \& Chevalier, C. (2019). Innovative Mucosal Vaccine Formulations Against Influenza A Virus Infections. Front Immunol, 10 , 1605. doi: 10.3389/fimmu.2019.01605

Chang, B. Y., Kim, S. B., Lee, M. K., Park, H., \& Kim, S. Y. (2015). Improved Chemotherapeutic Activity by Morus alba Fruits through Immune Response of Toll-Like Receptor 4.Int J Mol Sci, 16 (10), 24139-24158. doi: 10.3390/ijms161024139

Chang, B. Y., Kim, S. B., Lee, M. K., Park, H., \& Kim, S. Y. (2016). Nonclinical Safety Assessment of Morus alba L. Fruits: Study of 90-D Toxicity in Sprague Dawley Rats and Genotoxicity in Salmonella. $J$ Food Sci, 81 (5), T1328-1335. doi: 10.1111/1750-3841.13285

Cribbs, D. H., Ghochikyan, A., Vasilevko, V., Tran, M., Petrushina, I., Sadzikava, N., . . Agadjanyan, M. G. (2003). Adjuvant-dependent modulation of Th1 and Th2 responses to immunization with beta-amyloid. Int Immunol, 15 (4), 505-514.

Curtis, M. J., Alexander, S., Cirino, G., Docherty, J. R., George, C. H., Giembycz, M. A., . . . Ahluwalia, A. (2018). Experimental design and analysis and their reporting II: updated and simplified guidance for authors and peer reviewers. British Journal of Pharmacology, 175 (7), 987-993. doi: 10.1111/bph.14153

Ghorpade, D. S., Holla, S., Sinha, A. Y., Alagesan, S. K., \& Balaji, K. N. (2013). Nitric oxide and KLF4 protein epigenetically modify class II transactivator to repress major histocompatibility complex II expression during Mycobacterium bovis bacillus Calmette-Guerin infection. J Biol Chem, 288 (28), 20592-20606. doi: 10.1074/jbc.M113.472183

Goff, P. H., Hayashi, T., He, W., Yao, S., Cottam, H. B., Tan, G. S., . . . Krammer, F. (2017). Synthetic Toll-Like Receptor 4 (TLR4) and TLR7 Ligands Work Additively via MyD88 To Induce Protective Antiviral Immunity in Mice. 91 (19). doi: 10.1128/jvi.01050-17

Gu, P., Wusiman, A., Wang, S., Zhang, Y., Liu, Z., Hu, Y., . . . Wang, D. (2019). Polyethyleniminecoated PLGA nanoparticles-encapsulated Angelica sinensis polysaccharide as an adjuvant to enhance immune responses. Carbohydr Polym, 223 , 115128. doi: 10.1016/j.carbpol.2019.115128

Harandi, A. M. (2018). Systems analysis of human vaccine adjuvants. Semin Immunol, 39 , 30-34. doi: 10.1016/j.smim.2018.08.001

Huber, V. C., McKeon, R. M., Brackin, M. N., Miller, L. A., Keating, R., Brown, S. A., . . . McCullers, J. A. (2006). Distinct contributions of vaccine-induced immunoglobulin G1 (IgG1) and IgG2a antibodies to protective immunity against influenza. Clin Vaccine Immunol, 13 (9), 981-990. doi: 10.1128/cvi.00156-06

Hwang, K. H., \& Kim, Y. K. (2004). Promoting effect and recovery activity from physical stress of the fruit of Morus alba. Biofactors, 21 (1-4), 267-271.

Jahanban-Esfahlan, R., Seidi, K., \& Majidinia, M. (2019). Toll-like receptors as novel therapeutic targets for herpes simplex virus infection. 29 (4), e2048. doi: 10.1002/rmv.2048 
Jian, Y. R., Chang, S. Y., Lin, P. Y., Yang, Y. H., \& Chuang, Y. H. (2013). Inactivated influenza virus vaccine is efficient and reduces IL-4 and IL-6 in allergic asthma mice.Influenza Other Respir Viruses, 7 (6), 1210-1217. doi: 10.1111/irv.12150

Jones, D. E., Ackermann, M. R., Wille, U., Hunter, C. A., \& Scott, P. (2002). Early enhanced Th1 response after Leishmania amazonensis infection of C57BL/6 interleukin-10-deficient mice does not lead to resolution of infection.Infect Immun, 70 (4), 2151-2158.

Joshi, S. R., Shaw, A. C., \& Quagliarello, V. J. (2009). Pandemic influenza H1N1 2009, innate immunity, and the impact of immunosenescence on influenza vaccine. Yale J Biol Med, 82 (4), 143-151.

Kannan, S., Shaik Syed Ali, P., Sheeza, A., \& Hemalatha, K. (2020). COVID-19 (Novel Coronavirus 2019) - recent trends. Eur Rev Med Pharmacol Sci, 24 (4), 2006-2011. doi: 10.26355/eurrev_202002_20378

Kim, H. G., Ju, M. S., Shim, J. S., Kim, M. C., Lee, S. H., Huh, Y., . . . Oh, M. S. (2010). Mulberry fruit protects dopaminergic neurons in toxin-induced Parkinson's disease models. Br J Nutr, 104 (1), 8-16. doi: $10.1017 /$ S0007114510000218

Kim, S. B., Chang, B. Y., Hwang, B. Y., Kim, S. Y., \& Lee, M. K. (2014). Pyrrole alkaloids from the fruits of Morus alba. Bioorg Med Chem Lett, 24 (24), 5656-5659. doi: 10.1016/j.bmcl.2014.10.073

Kim, S. B., Chang, B. Y., Jo, Y. H., Lee, S. H., Han, S. B., Hwang, B. Y., . . . Lee, M. K. (2013). Macrophage activating activity of pyrrole alkaloids from Morus alba fruits. J Ethnopharmacol, 145 (1), 393-396. doi: $10.1016 /$ j.jep.2012.11.007

Kuznetsova, T. A., \& Persiyanova, E. V. (2019). [Adjuvants of influenza vaccines: new possibilities of using sulphated polysaccharides from marine brown algae.].64 (1), 5-11. doi: 10.18821/0507-4088-2019-64-1-5-11

Liu, K. S., Zhang, C., Dong, H. L., Li, K. K., Han, Q. B., Wan, Y., . . . Han, X. Q. (2019). GSP-2, a polysaccharide extracted from Ganoderma sinense, is a novel toll-like receptor 4 agonist. 14 (8), e0221636. doi: $10.1371 /$ journal.pone.0221636

Martinon, S., Cisneros, A., \& Villicana, S. (2019). Chemical and Immunological Characteristics of Aluminum-Based, Oil-Water Emulsion, and Bacterial-Origin Adjuvants.2019 , 3974127. doi: $10.1155 / 2019 / 3974127$

Medvedev, A. E. (2013). Toll-like receptor polymorphisms, inflammatory and infectious diseases, allergies, and cancer. J Interferon Cytokine Res, 33 (9), 467-484. doi: 10.1089/jir.2012.0140

Mohan, T., Zhu, W., Wang, Y., \& Wang, B. Z. (2018). Applications of chemokines as adjuvants for vaccine immunotherapy. Immunobiology, 223 (6-7), 477-485. doi: 10.1016/j.imbio.2017.12.001

Munoz, V. L., Porsch, E. A., \& St Geme, J. W., 3rd. (2019). Kingella kingae Surface Polysaccharides Promote Resistance to Neutrophil Phagocytosis and Killing. MBio, 10 (3). doi: 10.1128/mBio.00631-19

Popov, S. V., Golovchenko, V. V., Ovodova, R. G., Smirnov, V. V., Khramova, D. S., Popova, G. Y., \& Ovodov, Y. S. (2006). Characterisation of the oral adjuvant effect of lemnan, a pectic polysaccharide of Lemna minor L. Vaccine, 24 (26), 5413-5419.

Roy, R., Kumar, S., Verma, A. K., Sharma, A., Chaudhari, B. P., Tripathi, A., . . . Dwivedi, P. D. (2014). Zinc oxide nanoparticles provide an adjuvant effect to ovalbumin via a Th2 response in Balb/c mice. Int Immunol, 26 (3), 159-172. doi: 10.1093/intimm/dxt053

Rydell, N., \& Sjoholm, I. (2004). Oral vaccination against diphtheria using polyacryl starch microparticles as adjuvant. Vaccine, 22 (9-10), 1265-1274. doi: 10.1016/j.vaccine.2003.09.034

Singhal, T. (2020). A Review of Coronavirus Disease-2019 (COVID-19). Indian J Pediatr, 87 (4), 281-286. doi: $10.1007 / \mathrm{s} 12098-020-03263-6$ 
Terrinoni, M., Holmgren, J., Lebens, M., \& Larena, M. (2019). Requirement for Cyclic AMP/Protein Kinase A-Dependent Canonical NFkappaB Signaling in the Adjuvant Action of Cholera Toxin and Its Non-toxic Derivative mmCT. Front Immunol, 10 , 269. doi: 10.3389/fimmu.2019.00269

Tian, X., \& Liang, T. (2019). Extraction, Structural Characterization, and Biological Functions of Lycium Barbarum Polysaccharides: A Review. 9 (9). doi: 10.3390/biom9090389

Tuo, W., Palmer, G. H., McGuire, T. C., Zhu, D., \& Brown, W. C. (2000). Interleukin-12 as an adjuvant promotes immunoglobulin $\mathrm{G}$ and type 1 cytokine recall responses to major surface protein 2 of the ehrlichial pathogen Anaplasma marginale.Infect Immun, 68 (1), 270-280. doi: 10.1128/iai.68.1.270-280.2000

Wusiman, A., Gu, P., Liu, Z., Xu, S., Zhang, Y., Hu, Y., . . . Huang, X. (2019). Cationic polymer modified PLGA nanoparticles encapsulating Alhagi honey polysaccharides as a vaccine delivery system for ovalbumin to improve immune responses.Int J Nanomedicine, 14 , 3221-3234. doi: 10.2147/ijn.s203072

Xu, H. S., Wu, Y. W., Xu, S. F., Sun, H. X., Chen, F. Y., \& Yao, L. (2009). Antitumor and immunomodulatory activity of polysaccharides from the roots of Actinidia eriantha.J Ethnopharmacol, 125 (2), 310-317. doi: 10.1016/j.jep.2009.06.015

Yanase, N., Toyota, H., Hata, K., Yagyu, S., Seki, T., Harada, M., . . . Mizuguchi, J. (2014). OVA-bound nanoparticles induce OVA-specific IgG1, IgG2a, and IgG2b responses with low IgE synthesis. Vaccine, 32 (45), 5918-5924. doi: 10.1016/j.vaccine.2014.08.059

Yang, L., Tian, D., \& Liu, W. (2020). [Strategies for vaccine development of COVID-19].Sheng Wu Gong Cheng Xue Bao, 36 (4), 593-604. doi: 10.13345/j.cjb.200094

Yang, X. Y., Park, G. S., Lee, M. H., Chang, I. A., Kim, Y. C., Kim, S. Y., . . . Park, H. (2009). Toll-like receptor 4-mediated immunoregulation by the aqueous extract of Mori Fructus. Phytother Res, 23 (12), 1713-1720. doi: 10.1002/ptr.2818

(A)

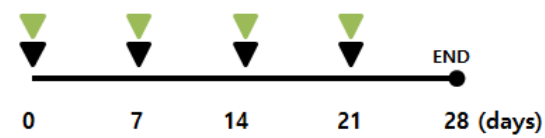

Ovalbumin(p.o.)

4 Morus alba L., CT (p.o.)

(B)

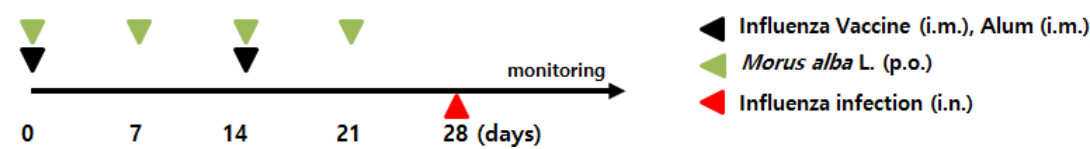


(A)

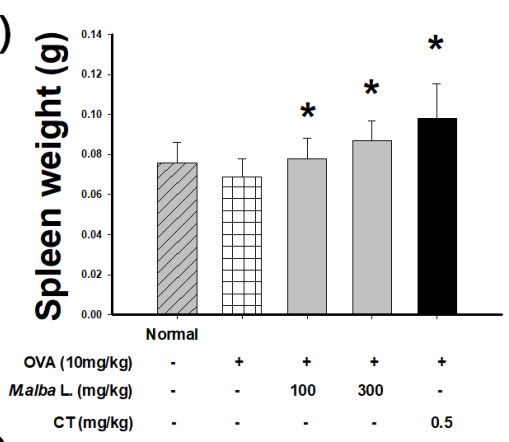

(C)

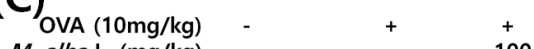

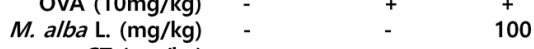
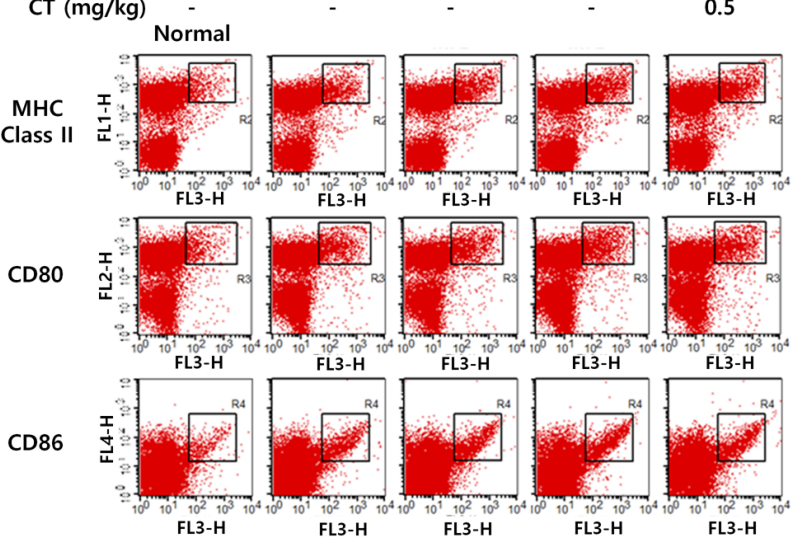

(B)

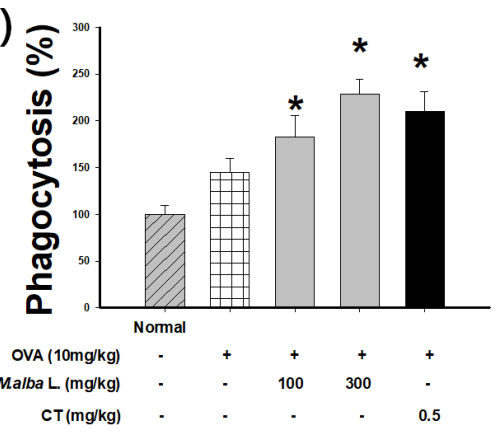

(A)

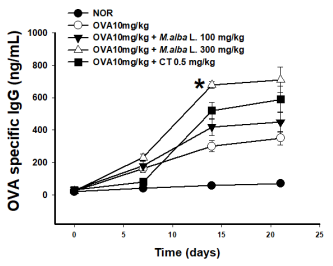

(B)

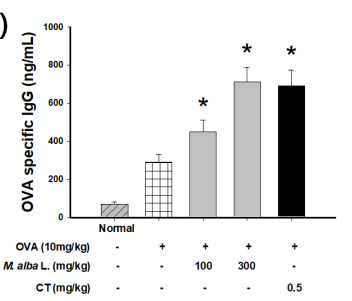

(C) $\overrightarrow{\mathrm{E}}$

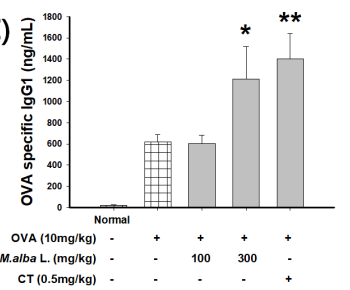

(F)

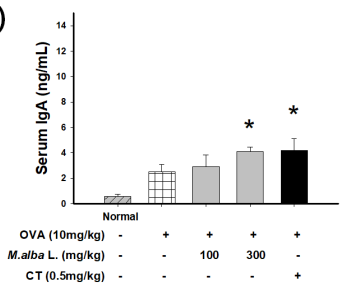

(D)

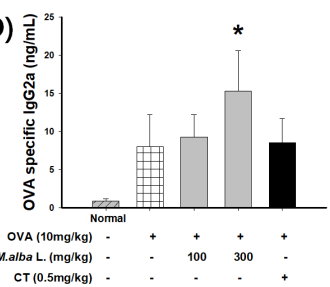

(G)

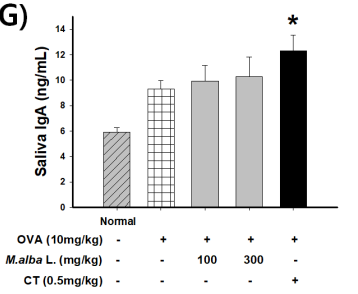

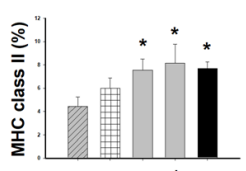
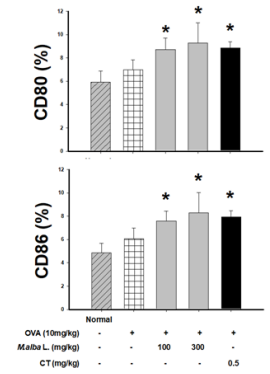

TT $(0.5 \mathrm{mg} / \mathrm{kg})$.

CT $(0.5 \mathrm{mg} / \mathrm{kg})$
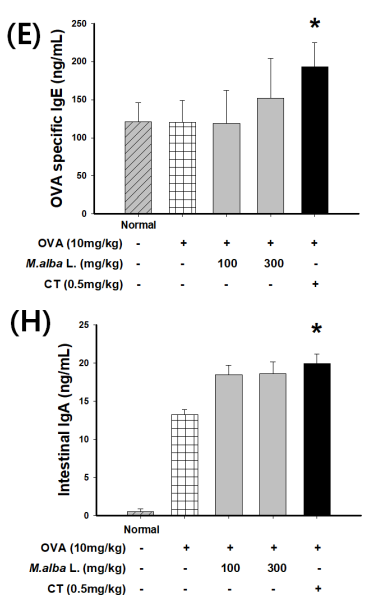

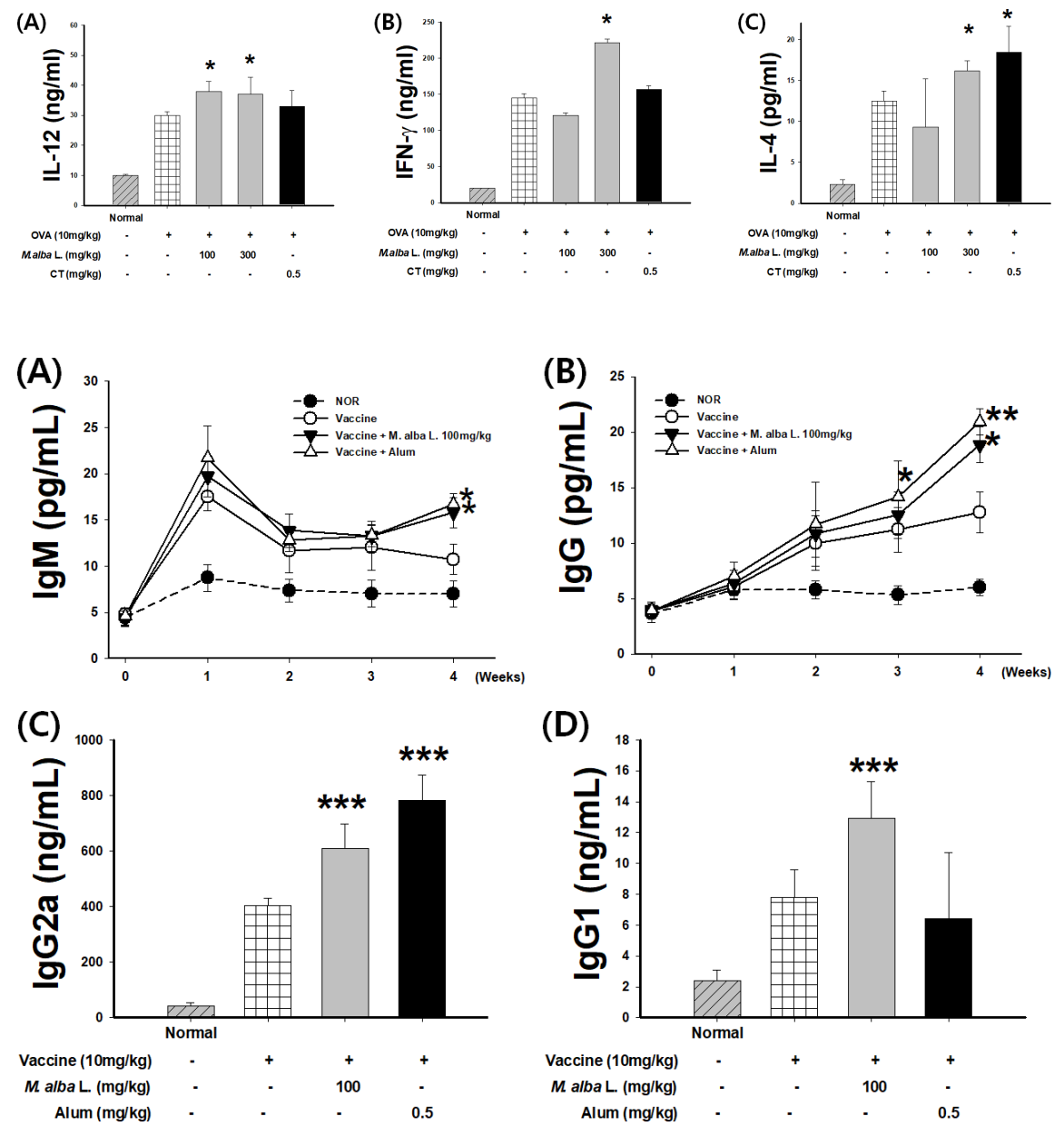

(A)

(B)
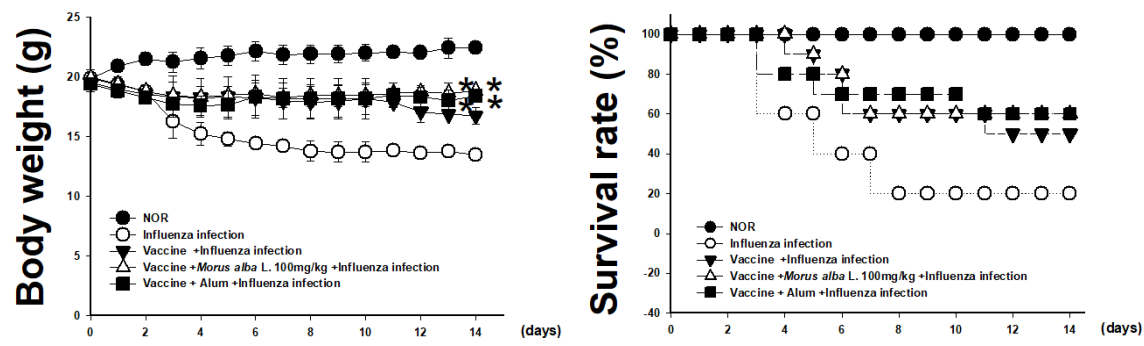